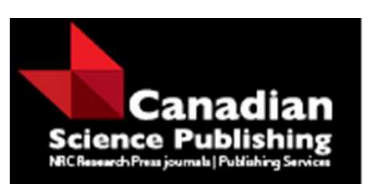

Canadian Journal of Physics

Revue canadienne de physique

\title{
Locally rotationally symmetric Bianchi type I massive string cosmological model with vacuum energy density and magnetic field in general relativity
}

\begin{tabular}{|r|l|}
\hline Journal: & Canadian Journal of Physics \\
\hline Manuscript ID & cjp-2015-0691 \\
\hline Manuscript Type: & Article \\
\hline Date Submitted by the Author: & 31 -Oct-2015 \\
\hline Complete List of Authors: & $\begin{array}{l}\text { Bali, Raj; University of Rajasthan, Mathematics } \\
\text { Singh, Swati; University of Rajasthan, Department of Mathematics }\end{array}$ \\
\hline Keyword: & LRS, Bianchi I, Massive string, Vacuum energy, Cosmological \\
\hline \multicolumn{2}{|l}{} \\
\hline
\end{tabular}

\section{SCHOLARONE \\ Manuscripts}




\title{
Locally rotationally symmetric Bianchi type I massive string cosmological model with vacuum energy density and magnetic field in general relativity
}

\author{
Raj Bali* and Swati Singh** \\ *CSIR Emeritus Scientist, **Research Associate in CSIR Major Project \\ Department of Mathematics, University of Rajasthan, Jaipur-302004, India
}

\begin{abstract}
\end{abstract}
Locally rotationally symmetric (LRS) Bianchi Type I massive string cosmological model with vacuum energy density $(\Lambda)$ and magnetic field is investigated. To get the deterministic model of universe, we assume that shear $(\sigma)$ is proportional to expansion $(\theta)$ and $\Lambda \sim \frac{1}{\mathrm{R}^{2}}$ as considered by Chen and Wu (Phys. Rev. 041, 695 (1990)) where $\mathrm{R}$ is scale factor. We find that the total energy density ( $\rho)$, the particle density $\rho_{p}$ decreases with time. The strong energy conditions as given by Hawking and Ellis [35] are satisfied. The vacuum energy density decreases with time which matches with astronomical observation. The model in general represents anisotropic space-time due to the presence of string. The total energy density and string tension density decrease due to the presence of magnetic field. Both the model have Point Type singularities at $\mathrm{T}=0$ and $\tau=0$ respectively. The other physical aspects of the models are also discussed.

Keywords : LRS, Bianchi I, Massive string, Cosmological, Vacuum energy, Magnetic field.

\section{Introduction}

The cosmological problem within the frame work of general relativity is to find a model of physical universe which correctly predicts the result of 
astronomical observation. The simplest models of expanding universe are those which are spatially homogeneous and isotropic at each instant of time i.e.

Corresponding author : Raj Bali (E-mail: balir5@yahoo.co.in

Friedmann-Robertson-Walker (FRW) models. But FRW models are unstable near the singularity (Patridge and Wilkinson [1] and fail to describe early universe. Therefore spatially homogeneous and anisotropic Bianchi models are undertaken to study the universe in its early stages of evolution. Recent cosmological observations support the existence of anisotropic universe that approaches the isotropic phase (Land and Magueijo [2]. For simplification and description of large scale behaviour of the actual universe, LRS Bianchi models have great importance. Lidsey [3] investigated that these models are equivalent to FRW models which are considered standard models of our universe.

The universe is represented by collection of extended objects, called galaxies (Letelier [4]. The existence of string in the early universe can be explained using grand unified field theories as given by Kibble [5] and Vilenkin [6]. These strings have stress energy and coupled with the gravitational field. Therefore it is interesting to study the gravitational effects that arise from strings. The pioneering work in the formulation of energy-momentum tensor for classical massive strings was initiated by Letelier [7] who explained that massive strings are formed by geometric string with particles attached along its extension and used this idea to find string cosmological solutions using Bianchi Type I and Kantowski-Sach's space-time. Melvin [8] in his investigation for cosmological solution for dust and electromagnetic field suggested that during the evolution of the universe, the matter was in highly ionized state and is smoothly coupled with 
the gravitational field. Hence the presence of magnetic field in string dust universe is not unrealistic. Therefore, it is reasonable to consider magnetic field in the energy-momentum tensor of early universe as the inclusion of magnetic field is supported by observational cosmology and astrophysics. A cosmological model containing global magnetic field is necessarily anisotropic. The present observed universe is almost isotropic at large, therefore, isotropization problem appears in any study of anisotropic cosmologies. String cosmological models have been used in attempts to investigate the anisotropic behaviour of early universe. Therefore, we attempt to investigate the effect of magnetic field during early evolution of universe in string cosmological models. Many authors viz. Banerjee et al. [9], Tikekar and Patel [10,11], Wang [12], Bali et al. [13,14], Saha et al. [15],Singh [16] investigated string cosmological models with magnetic field using Letelier [7] concept with magnetic field.

The current accelerating expansion of the universe suggests that our universe is dominated by unknown dark energy. The cosmological constant is the most favoured candidate of dark energy representing energy density of vacuum cosmological constant occupies a privileged place in the dark energy models because it provides a good approximation to the present astronomical data as studied by Zel'dovich [17], Dreitlein [18], Krauss and Turner [19]. In continuation of the study, two independent groups led by Riess et al. [20] and Perlmutter et al. [21] used Type Ia supernovae to show that universe is not only expanding but also accelerating. This discovery provided that the cosmological constant $(\Lambda)$ is nonzero. Now, it is believed that the cosmological constant is a kind of repulsive 
pressure dubbed as dark energy, is the most suitable candidate to explain the recent observation of universe. Many authors viz. Bertolami [22], Chen and $\mathrm{Wu}$ [23], Sahni and Starobinsky [24], Peebles and Ratra [25], Singh and Kumar [26], Bali and Singh $[27,28]$ investigated cosmological models with decaying vacuum energy density.

\section{Metric and Field Equations}

We consider LRS Bianchi Type I Space-time in the form

$$
d s^{2}=-d t^{2}+A^{2} d x^{2}+B^{2}\left(d y^{2}+d z^{2}\right)
$$

where $\mathrm{A}$ and $\mathrm{B}$ are metric potentials and are functions of t-alone. The energymomentum tensor for a cloud of string is given by Letelier [7] and Lichnerowicz [29] as

$$
T_{i}^{j}=\rho v_{i} v^{j}-\lambda x_{i} x^{j}+E_{i}^{j}
$$

where $v^{i}$ and $x^{i}$ satisfy the conditions

$$
\begin{aligned}
& v_{i} v^{i}=-x_{i} x^{i}=-1, v^{i} x_{i}=0 \\
& x_{1} \neq 0, x_{2}=0=x_{3}=x_{4}
\end{aligned}
$$

$\rho$ being the proper energy density for cloud of string with particles attached to them, $\lambda$ the string tension density, $v^{i}$ the four velocity of particles and $x^{i}$ the unit space-like vector representing the direction of string. If the particle density of the configuration is denoted by $\rho_{\mathrm{p}}$ then for massive string, we have

$$
\rho=\rho_{p}+\lambda
$$

In a comoving coordinate system, we have 


$$
v^{i}=(0,0,0,1), x^{i}=\left(\frac{1}{A}, 0,0,0\right)
$$

$\mathrm{E}_{\mathrm{i}}^{\mathrm{j}}$ is the electromagnetic field tensor given by Lichnerowicz [29] as

$$
E_{i}^{j}=\bar{\mu}\left[|h|^{2}\left(v_{i} v^{j}+\frac{1}{2} g_{i}^{j}\right)-h_{i} h^{j}\right]
$$

with

$$
\mathrm{h}_{\mathrm{i}}=\frac{\sqrt{-\mathrm{g}}}{2 \bar{\mu}} \in \in_{\mathrm{ijk} \ell} \mathrm{F}^{\mathrm{k} \ell} \mathrm{v}^{\mathrm{j}}
$$

where $h_{i}$ is the magnetic flux vector, $\epsilon_{i j k \ell}$ the Levi-Civita tensor, $F^{k \ell}$ the electromagnetic field tensor, $\bar{\mu}$ the magnetic permeability $|\mathrm{h}|^{2}=\mathrm{h}_{\mathrm{i}} \mathrm{h}^{\mathrm{i}}$ and $\mathrm{g}_{\mathrm{ij}}$ the metric tensor. We assume that the magnetic field is due to an electric current produced along $\mathrm{x}$-axis. Thus $\mathrm{F}_{23}$ is the only non-vanishing component of $\mathrm{F}_{\mathrm{ij}}$ and $\mathrm{h}_{1} \neq 0, \mathrm{~h}_{2}=0=\mathrm{h}_{3}=\mathrm{h}_{4}$. Maxwell's equations $\mathrm{F}_{[\mathrm{ij} ; \mathrm{k}]}=0$ and $\mathrm{F}_{; \mathrm{j}}^{\mathrm{ij}}=0$ are satisfied by

$$
\mathrm{F}_{23}=\mathrm{H}(\text { constant })
$$

We also fund that $\mathrm{F}_{14}=0=\mathrm{F}_{24}=\mathrm{F}_{34}$ due to the assumption of infinite electrical conductivity. Equation (7) leads to

$$
\mathrm{h}_{1}=\frac{\mathrm{AH}}{\bar{\mu} \mathrm{B}^{2}}
$$

and equations (9) and (6) lead to

$$
E_{1}^{1}=-\frac{H^{2}}{2 \bar{\mu} B^{4}}=-E_{2}^{2}=-E_{3}^{3}=E_{4}^{4}
$$

Einstein's field equations 


$$
\mathrm{R}_{\mathrm{i}}^{\mathrm{j}}-\frac{1}{2} \mathrm{Rg}_{\mathrm{i}}^{\mathrm{j}}-\Lambda \mathrm{g}_{\mathrm{i}}^{\mathrm{j}}=-\mathrm{T}_{\mathrm{i}}^{\mathrm{j}}
$$

(in geometrical units where $8 \pi \mathrm{G}=1=\mathrm{c}$ )

for the line-element (1) lead to

$$
\begin{aligned}
& \frac{2 B_{44}}{B}+\frac{B_{4}^{2}}{B^{2}}=\Lambda+\lambda+\frac{k}{B^{4}} \\
& \frac{A_{44}}{A}+\frac{B_{44}}{B}+\frac{A_{4} B_{4}}{A B}=\Lambda-\frac{k}{B^{4}} \\
& \frac{2 A_{4} B_{4}}{A B}+\frac{B_{4}^{2}}{B^{2}}=\rho+\Lambda+\frac{k}{B^{4}}
\end{aligned}
$$

where

$$
\mathrm{k}=\frac{\mathrm{H}^{2}}{2 \bar{\mu}}
$$

\section{Solution of field equations}

To get the deterministic solution in terms of cosmic time $t$, we assume that shear $(\sigma)$ is proportional to expansion $(\theta)$ as considered by Thorne [30]. Thus, we have

$$
\mathrm{A}=\mathrm{B}^{\mathrm{n}}
$$

where $\mathrm{A}$ and $\mathrm{B}$ are metric potentials and $\mathrm{n}$ is a constant. The motive for assuming the condition $\sigma \alpha \theta$ is explained as : Referring to Thorne [30], the observations of the velocity- red shift relation for extra galactic sources suggest that the Hubble expansion of the universe is isotropic within 30 percent (Kantowski and Sachs [31], Kristian and Sachs [32]). More precisely, the red-shift studies plate the limit $\frac{\sigma}{\mathrm{H}} \leq 0.30$ where $\sigma$ is shear and $\mathrm{H}$ is Hubble constant. Also Collins et al. [33] have 
pointed out that for spatially homogeneous metric, the normal congruence to the homogeneous hypersurface satisfies the condition $\frac{\sigma}{\theta}=$ constant.

Equations (13) and (16) lead to

$$
(\mathrm{n}+1) \frac{\mathrm{B}_{44}}{\mathrm{~B}}+\mathrm{n}^{2} \frac{\mathrm{B}_{4}^{2}}{\mathrm{~B}^{2}}=\Lambda-\frac{\mathrm{K}}{\mathrm{B}^{4}}
$$

To get the deterministic model, we assume that $\Lambda=\frac{\alpha}{\mathrm{R}^{2}}$ as assumed by Chen and $\mathrm{Wu}[23]$ where $\mathrm{R}$ is scale factor. Thus, we have

$$
\Lambda=\alpha B^{-\frac{2(n+2)}{3}}
$$

Equations (17) and (18) lead to

$$
5 \frac{\mathrm{B}_{44}}{\mathrm{~B}}+16 \frac{\mathrm{B}_{4}^{2}}{\mathrm{~B}^{2}}=\frac{\alpha-\mathrm{K}}{\mathrm{B}^{4}}
$$

where we have assumed $n=4$ for deterministic model of universe. Equation (19) leads to

$$
\left(\frac{\mathrm{dB}}{\mathrm{dt}}\right)^{2}=\frac{\mathrm{b}+\mathrm{a} \mathrm{B}}{\mathrm{B}^{2}}
$$

where $b=\frac{\alpha-K}{11}$ and $a$ is constant of integration. After suitable transformation of coordinates, the metric (1) leads to the form

$$
\mathrm{ds}^{2}=-\frac{\mathrm{T}^{2} \mathrm{dT}^{2}}{\mathrm{~b}+\mathrm{aT}} \mathrm{T}^{-22 / 5}+\mathrm{T}^{8} \mathrm{dX^{2 }}+\mathrm{T}^{2}\left(\mathrm{dy}^{2}+\mathrm{dz}^{2}\right)
$$

where $\mathrm{B}=\mathrm{T}, \mathrm{x}=\mathrm{X}, \mathrm{y}=\mathrm{Y}, \mathrm{z}=\mathrm{Z}$ and cosmic time $\mathrm{t}$ is defined as

$$
t=\int \frac{T d T}{\sqrt{b+a T^{-22 / 5}}}
$$




\section{Special Case}

In absence of magnetic field and vacuum energy density i.e. $\mathrm{k}=0, \Lambda=0$, equation (19) leads to

$$
\frac{\mathrm{B}_{44}}{\mathrm{~B}_{4}}=-\frac{16}{5} \frac{\mathrm{B}_{4}}{\mathrm{~B}}
$$

which leads to

$$
\mathrm{B}_{4}=\ell \mathrm{B}^{-\frac{16}{5}}
$$

Thus we have

$$
\mathrm{B}^{\frac{21}{5}}=\frac{21 \ell}{5} \mathrm{t}+\mathrm{M}
$$

and

$$
A=B^{4}=\left(\frac{21 \ell}{5} t+M\right)^{\frac{20}{21}}=(L t+M)^{\frac{20}{21}}
$$

where $\mathrm{L}=\frac{21 \ell}{5}$ and $\mathrm{M}$ is constant of integration.

After using suitable transformation of coordinates, the metric (1) leads to the form

$$
d s^{2}=-\frac{d \tau^{2}}{a^{2}}+\tau^{\frac{40}{21}} d X^{2}+\tau^{\frac{10}{21}}\left(d Y^{2}+d Z^{2}\right)
$$

where $L t+M=\tau, x=X, y=Y, z=Z$.

For $\mathrm{L}=1, \mathrm{M}=0$, the metric (27) leads to

$$
d s^{2}=-d t^{2}+t^{\frac{40}{21}} d x^{2}+t^{\frac{10}{21}}\left(d y^{2}+d z^{2}\right)
$$




\section{Physical and geometrical features}

The total energy density $(\rho+\Lambda)$, vacuum energy density $(\Lambda)$, the string tension density $(\lambda)$, the particle density $\left(\rho_{p}\right)$, the spatial volume $\left(R^{3}\right)$, the expansion $(\theta)$, the Hubble parameter $(H)$, the shear $(\sigma)$, the deceleration parameters $(q)$ for the models (21) are given by

$$
\begin{aligned}
& \rho+\Lambda=(9 b-k) T^{-4}+9 a T^{-42 / 5} \\
& \Lambda=\frac{1}{\mathrm{~T}^{4}} \\
& \lambda=-\mathrm{bT}^{-4}-\frac{27 \mathrm{a}}{5} \mathrm{~T}^{-\frac{42}{5}}-\alpha \mathrm{T}^{-4}-\mathrm{kT}^{-4} \\
& \rho_{\mathrm{p}}=10 \mathrm{bT}^{-4}+\frac{72 \mathrm{a}}{5} \mathrm{~T}^{-\frac{42}{5}} \\
& \mathrm{R}^{3}=\mathrm{AB}^{2}=\mathrm{T}^{6} \\
& \theta=\frac{\dot{\mathrm{A}}}{\mathrm{A}}+2 \frac{\dot{\mathrm{B}}}{\mathrm{B}}=\sqrt{\mathrm{b}+\mathrm{aT}^{-22 / 5}} \\
& \mathrm{H}=\frac{\theta}{3}=\frac{2}{\mathrm{~T}^{2}} \sqrt{\mathrm{b}+\mathrm{a} \mathrm{T}^{-22 / 5}} \\
& \sigma=\frac{1}{\sqrt{3}}\left|\frac{\dot{\mathrm{A}}}{\mathrm{A}}-\frac{\dot{\mathrm{B}}}{\mathrm{B}}\right| \\
& =\frac{\sqrt{3}}{\mathrm{~T}^{2}} \sqrt{\mathrm{b}+\mathrm{aT}} \mathrm{T}^{-22 / 5} \\
& \frac{\sigma}{\theta}=\frac{1}{2 \sqrt{3}} \neq 0 \\
& \mathrm{q}=-1-\frac{\dot{\mathrm{H}}}{\mathrm{H}^{2}}
\end{aligned}
$$




$$
=-1+\frac{\sqrt{b T^{22 / 5}+a}}{10 B^{22 / 10}}\left[\frac{b^{22 / 5}+(10 a+11) T^{11 / 5}}{\left(b T^{33 / 5}+a\right)}\right]
$$

In absence of magnetic field $(\mathrm{k}=0)$ and vacuum energy density $(\Lambda=0)$, the above mentioned quantities are given by

$$
\begin{aligned}
\rho & =\frac{225 \mathrm{~L}^{2}}{441 \tau^{2}} \\
\lambda & =-\frac{135 \mathrm{~L}^{2}}{441 \tau^{2}} \\
\rho_{\mathrm{p}} & =\rho-\lambda=\frac{360 \mathrm{~L}^{2}}{441 \tau^{2}} \\
\mathrm{R} & =\mathrm{AB}=\tau^{30 / 21} \\
\theta & =\frac{\mathrm{A}}{\mathrm{A}}+\frac{2 \mathrm{~B}}{\mathrm{~B}}=\frac{10 \mathrm{~L}}{7 \tau} \\
\mathrm{H} & =\frac{\theta}{3} \\
& =\frac{1}{20}>0 \\
\sigma & =\frac{1-\frac{\dot{H}}{\mathrm{H}^{2}}}{21 \tau} \\
& =\frac{10 \mathrm{~L}}{\sqrt{3}}\left|\frac{\dot{A}}{\mathrm{~A}}-\frac{\dot{B}}{\mathrm{~B}}\right|
\end{aligned}
$$




\section{State Finder Parameters}

The state finder parameters effectively differentiate between different forms of dark energy and provide simple diagnosis whether a particular model fits into the basic observational data. Following Sahni et al. [34], the state finder diagnostic pair $\{\mathrm{r}, \mathrm{s}\}$ is given by

$$
\mathrm{r}=1+3 \frac{\dot{\mathrm{H}}}{\mathrm{H}^{2}}+\frac{\ddot{\mathrm{H}}}{\mathrm{H}^{3}}
$$

and

$$
\mathrm{s}=\frac{\mathrm{r}-1}{3\left(\mathrm{q}-\frac{1}{2}\right)}
$$

For the model (28), the state finder parameters $\{\mathrm{r}, \mathrm{s}\}$ are given by

$$
\mathrm{r}=3.52
$$

and

$$
\mathrm{s}=1.01
$$

We observe that $r>1$ and $s>1$.

\section{Discussion and Conclusion}

The strong energy condition $\rho \geq 0, \lambda<0$ as given by Hawking and Ellis [35] are satisfied for both the models (21) and (27). The spatial volume increases with time for both the models. The anisotropy is maintained for both the models due to the presence of string. Both the models represent decelerating phase of universe. However, the model (21) represents accelerating phase of universe at late time. Both the models start with a big-bang at $\mathrm{T}=0$ and $\tau=0$ respectively and expansion decreases with time. The total energy $(\rho+\Lambda)>0$ for the model (21) is 
satisfied when $b>k / 9$. Both the models (21) and (27) have Point Type singularity at $\mathrm{T}=0$ and $\tau=0$ respectively as given by MacCallum [36]. The state finder parameters are found to be $r>1$ and $s>1$ for the model (27).

\section{References}

1. R.B. Patridge and D.T. Wilkinson. Phys. Rev. Lett. 18. 557 (1967).

2. K. Land and J. Magueijo. Phys. Rev. Lett. 95. 071301 (2005).

3. J.E. Lidsey. Class. Quant. Gravity. 9. 1239 (1992).

4. $\quad$ P.S. Letelier. Phys. Rev. D20. 1294 (1979).

5. T.W.B. Kibble. J. Phys. A 9. 1387 (1976).

6. A. Vilenkin. Phys. Rev. Lett. 46. 1169 (1981).

7. P.S. Letelier. Phys. Rev. D28. 2414 (1983).

8. M.A. Melvin. Ann. New York Acad. Sci. 262. 253 (1975).

9. A. Banerjee, A.K. Sanyal and S. Chakravorty. Pramana - J. Phys. 34.1 (1990).

10. R. Tikekar and L.K. Patel. Gen. Relativ. Gravit. 24. 397 (1992).

11. R. Tikekar and L.K. Patel. Pramana - J. Phys. 42. 483 (1994).

12. X.X. Wang. Chin. Phys. Lett. 23. 1702 (2006).

13. R. Bali and Anjali. Astrophys. and Space-Science. 302. 201 (2006).

14. R. Bali, U. Pareek and A. Pradhan. Chin. Phys. Lett. 24. 2455 (2007).

15. B. Saha, M. Rikhvitsky and M. Visinescu. Cent. Eur. J. Phys. 8. 113 (2010).

16. C.P. Singh. Int. J. Theor. Phys.53. 1533 (2014).

17. Ya. B. Zel'dovich. Sov. Phys. 11. article 381 (1968).

18. J. Dreitlein. Phys. Rev. Lett. 33. 1243 (1974). 
19. L.M. Krauss and M.S.Turner. Gen. Relativ. Gravit. 27. 1157 (1995).

20. A.G.Riess et al. : Astron. J. 116.1009 (1998).

21. S. Perlmutter et al. Astrophys. J. 517. 565 (1999).

22. D. Bertolami. Nuovocim. B 93. 36 (1986).

23. W.Chen and Y.S. Wu. Phys. Rev. 041. 695 (1990).

24. V. Sahni and A.Starobinsky. Int. J. Mod. Phys. D 9. 373 (2000).

25. P.J.E. Peebles and B. Ratra. Rev. Mod. Phys. 75. 559 (2003).

26. C.P. Singh and S. Kumar. Int. J. Theor. Phys. 47.3171 (2008).

27. R. Bali and J.P. Singh. Int. J. Theor. Phys. 47. 3288 (2008).

28. R. Bali and S. Singh. Int. J. Theor. Phys. 53. 2082 (2014).

29. A. Lichnerowicz. Relativistic Hydrodynamics and Magnetohydrodynamics, Benjamin, New York, p. 13 (1967).

30. K.S. Thorne. Astrophys. J. 148. 51 (1967).

31. R. Kantowski and R.K. Sachs. J.Math. Phys. 7. 443(1966).

32. J. Kristian and R.K. Sachs. J. Math.Phys 143. 379 (1966).

33. C.B. Collins, E.N. Glass and D.A. Wilkinson. Gen. Relativ. Gravit. 12. 805 (1980).

34. V. Sahni, T.D. Saini, A.A.Starobinsky and U. Alam. JETP Letters. 77. 201 (2003).

35. S.W. Hawking and G.F.R. Ellis. The large scale structure of space-time, Cambridge, England, p. 88 (1974).

36. M.A.H. MacCallum. Comm. Math. Phys. 20. 57 (1971). 